\title{
Supersymmetric Representations and Integrable Fermionic Extensions of the Burgers and Boussinesq Equations
}

\author{
Arthemy V. KISELEV ${ }^{\dagger \ddagger}$ and Thomas WOLF $\S$ \\ † Department of Higher Mathematics, Ivanovo State Power University, \\ 34 Rabfakovskaya Str., Ivanovo, 153003 Russia \\ $¥$ Department of Physics, Middle East Technical University, 06531 Ankara, Turkey \\ E-mail: arthemy@newton.physics.metu.edu.tr \\ $\S$ Department of Mathematics, Brock University, 500 Glenridge Ave., \\ St. Catharines, Ontario, Canada L2S 3A1 \\ E-mail: twolf@brocku.ca
}

Received November 26, 2005, in final form February 25, 2006; Published online February 28, 2006

Original article is available at http://www.emis.de/journals/SIGMA/2006/Paper030/

\begin{abstract}
We construct new integrable coupled systems of $N=1$ supersymmetric equations and present integrable fermionic extensions of the Burgers and Boussinesq equations. Existence of infinitely many higher symmetries is demonstrated by the presence of recursion operators. Various algebraic methods are applied to the analysis of symmetries, conservation laws, recursion operators, and Hamiltonian structures. A fermionic extension of the Burgers equation is related with the Burgers flows on associative algebras. A Gardner's deformation is found for the bosonic super-field dispersionless Boussinesq equation, and unusual properties of a recursion operator for its Hamiltonian symmetries are described. Also, we construct a three-parametric supersymmetric system that incorporates the Boussinesq equation with dispersion and dissipation but never retracts to it for any values of the parameters.
\end{abstract}

Key words: integrable super-equations; fermionic extensions; Burgers equation; Boussinesq equation

2000 Mathematics Subject Classification: 35Q53; 37K05; 37K10; 37K35; 58A50; 81T40

\section{Introduction}

In this paper we construct new integrable coupled boson+fermion systems of $N=1$ supersymmetric equations and present fermionic extensions for the Burgers and Boussinesq equations. The integrability of new systems is established by finding weakly non-local $[2,14,22]$ recursion operators for their symmetry algebras or by describing Gardner's deformations [18, 6, 12]. We find three integrable $N=1$ supersymmetric analogues of the $\mathrm{KdV}$ equation. Also, we relate a fermionic extension of the Burgers equation with the Burgers equations on associative algebras [20]. We apply algebraic methods [9] to the study of geometry of supersymmetric PDE, and we use the SsTools package [8] for the computer algebra system REDUCE in practical computations.

First we deal with generalizations of the Burgers equation, which describes the dissipative nonlinear evolution of rarified gas. We consider the bosonic super-field version of the Burgers equation and construct two infinite sequences of its symmetries; the sequences correspond to even and odd 'times' along the flows. Next, we analyze a family of fermionic extensions for the Burgers equation itself. We show that if the coupling is zero, then the system at hand 
reduces to the Burgers super-equation w.r.t. a new field that unites the initial fermionic and bosonic components. Otherwise, a new Grassmann independent variable is introduced and the fermionic extensions are transformed to the Burgers equation on an associative algebra (see also [20]). Also, we observe that an $N=2$ generalization of the Burgers equation appears as a symmetry flow for the Laberge-Mathieu's $N=2$ supersymmetric $\mathrm{SKdV}_{4}$ equation [13]. The diagonal reduction $\theta^{1}=\theta^{2}$ of this Burgers system is the $N=1$ super-field Burgers equation.

Further, we consider the systems related with the Boussinesq equation, which describes the propagation of waves in a weakly nonlinear and weakly dispersive liquid. We construct a Gardner's deformation $[18,12]$ of the dispersionless bosonic super-field Boussinesq equation. Thus we recursively define its Hamiltonians, which are further transmitted to the boson+fermion representation of the system at hand. Independently, we construct an unusual weakly non-local [2, 22] recursion operator for this system: its differential order is 1, although it proliferates the symmetries of constant order 2. These symmetries are Hamiltonian w.r.t. the previously found chains of functionals, and the 'times' along the flows are even and odd variables, respectively. Finally, we extend a super-field representation for the 'full' Boussinesq equation with dispersion and dissipation to a family of coupled boson+fermion evolutionary super-systems that contain the Boussinesq equation but do not retract to it at any values of the parameters.

The paper is organized as follows. In the introductory part below, we describe three analogues of the $N=1$ supersymmetric $\mathrm{KdV}$ equation $[15,16]$ and construct weakly non-local recursion operators for their symmetry algebras, see Example 1 on p. 3 and Example 2 in Section 2. Also, we discuss the properties of a non-local Gardner's deformation $[1,18]$ for the $N=1$ supersymmetric KdV equation itself. In Section 2 we recall two schemes for generating infinite sequences of higher symmetries of the evolutionary super-systems which are contained in the experimental database [27] and which are the objects studied in this paper. In Section 3 we investigate super-field representations and fermionic extensions of the Burgers equation. Then in Section 4 we discuss a Gardner's deformation of the dispersionless Boussinesq equation, and we construct a parametric family of super-systems that incorporate the Boussinesq equation with dispersion and dissipation.

\subsection{The classification problem}

The motivating idea of this research is the problem of a complete description of $N=1$ supersymmetric nonlinear scaling-invariant evolutionary equations $\left\{f_{t}=\phi^{f}, b_{t}=\phi^{b}\right\}$ that admit infinitely many local symmetries proliferated by recursion operators; here $b(x, t, \theta)$ is the bosonic super-field and $f(x, t, \theta)$ is the fermionic super-field. We denote by $\theta$ the super-variable and we put $\mathcal{D} \equiv D_{\theta}+\theta D_{x}$ such that $\mathcal{D}^{2}=D_{x}$ and $[\mathcal{D}, \mathcal{D}]=2 D_{x}$; here $D_{\theta}$ and $D_{x}$ are the derivatives w.r.t. $\theta$ and $x$, respectively (fortunately, the derivative $D_{\theta}$ is met very seldom in the text, hence no confusion with the operator $\mathcal{D}$ occurs). The following axioms suggested by V.V. Sokolov and A.S. Sorin were postulated:

1. Each equation admits at least one higher symmetry $\left(\begin{array}{l}f_{s} \\ b_{s}\end{array}\right)$.

2. All equations are translation invariant and do not depend on the time $t$ explicitly.

3. None of the evolution equations involves only one field and hence none of the r.h.s. vanishes.

4. At least one of the right-hand sides in either the evolution equation or its symmetry is nonlinear.

5. At least one equation in a system or at least one component of its symmetry contains a fermionic field or the super-derivative $\mathcal{D}$.

6 . The time $t$ and the parameters $s$ along the integral trajectories of the symmetry fields are even variables (that is, the parities of $b$ and $b_{t}$ or $b_{s}$ coincide, as well as the parities of $f, f_{t}$, and $f_{s}$ ). 
7. The equations are scaling invariant: their right-hand sides are differential polynomials homogeneous w.r.t. a set of (half-)integer weights $[\theta] \equiv-\frac{1}{2},[x] \equiv-1,[t]<0,[f],[b]>0$; we also assume that the negative weight $[s]$ is (half-)integer.

Axiom 8 for $N \geq 2$ supersymmetric equations that satisfy the above axioms is further described on p. 14. If a particular equation under study admits symmetry flows with an odd 'time', then we use the notation $\bar{s}$ and the parities of $f_{\bar{s}}, b_{\bar{s}}$ are opposite to the parities of $f$ and $b$, respectively.

Remark 1. From Axiom 5 it follows that the admissible systems are either fermionic extensions of bosonic systems, or they are $N \geq 1$ supersymmetric super-field equations and the derivations $\mathcal{D}$ are present explicitly. For instance, the superKdV equation (2) w.r.t. a fermionic super-field $f(x, t, \theta)$ is $N=1$ supersymmetric, see $[15,16]$. On the other hand, an $N=0$ twocomponent fermionic extension of the Burgers equation that can not be represented as a scalar $N=1$ equation is obtained in Section 3.2, see equation (16) on p. 10.

The first version of the Reduce package SsTools by T. Wolf and W. Neun for supercalculus allowed to do symmetry investigations of supersymmetric equations. It was used for finding scalar fermionic and bosonic super-field and $N=1$ supersymmetric equations, and for description of coupled fermion+fermion, fermion+boson, and boson+boson evolutionary systems that satisfy the above axioms; a number of $N=2$ scalar evolution PDE were also found. The bounds $0<[f],[b] \leq 5$ and $0>[t]>[s] \geq-5$ were used. The experimental database [27] contains 1830 equations (the duplication of PDE that appeared owing to possible non-uniqueness of the weights is now eliminated) and their 4153 symmetries (plus the translations along $x$ and $t$, and plus the scalings whose number is in fact infinite).

Remark 2. Axiom 7 together with Axiom 3 are very restrictive. Indeed, a class of integrable systems that do not satisfy these two assumptions is provided by the Gardner's deformations, see [18] and [6, 12]. The extended $N=1 \operatorname{superKdV}_{\varepsilon}$ equation (3) and equation (27) on p. 15 give two examples; many other completely integrable extended systems are found in loc. cit. We emphasize that no scaling invariance can be recognized for the Gardner's extended systems if non-zero values of the deformation parameters $\varepsilon$ are fixed. The scaling invariance is restored if the weights of the parameters are assumed non-zero: one has $[\varepsilon]=-1$ for equation $(3)$ and $[\varepsilon]=-3$ for system (27). Hence we conclude that a classification (see [24] and references therein) of the 'symmetry integrable' homogeneous evolution equations may be incomplete, providing only zero order terms of the deformations in $\varepsilon$.

Also, we note that the symmetry integrability approach, which was used to fill in the database [27], has revealed a number of systems whose integrability in any sense remains an open problem. For example, supersymmetric equation (33) admits four symmetries, but this knowledge can hardly contribute to constructing a solution of the system at hand.

Equations (3) and (33) demonstrate that the database [27] with supersymmetric and coupled boson+fermion systems is not exhaustive and, simultaneously, it may contain equations whose complete or Lax integrability is uncertain.

\subsection{The $N=1$ superKdV equation and its extensions}

The classical integrable supersymmetric evolutionary systems as well as their generalizations and reductions are present in the database.

Example $1(N=1$ superKdV $[15,16])$. Let $N=1$ and let the weight of the bosonic super-field $b(x, t, \theta)$ be $[b]=1$; further, let $[t]=-3$. Now we scan the cell which is assigned to these weights and which is filled in by the runs of SsTools. Then we get the potential $N=1$ 
superKdV equation $^{1}$

$$
b_{t}=b_{x x x}+3 \mathcal{D}\left(b_{x} \mathcal{D} b\right) \text {. }
$$

Indeed, put $f(x, t ; \theta)=\mathcal{D} b$; then $f$ is the fermionic super-field of weight $[f]=\frac{3}{2}$ satisfying the superKdV equation $([15,16]$, see also [5] and $[9$, Ch. 6])

$$
f_{t}=f_{x x x}+3(f \mathcal{D} f)_{x} .
$$

The potential super-field equation (1) admits two conserved densities $\rho_{1}=b, \rho_{2}=\frac{1}{2} b^{2}$, which are nonlocal w.r.t. equation (2); also, potential equation (1) inherits infinitely many fermionic conservation laws from superKdV equation (2). The local conserved densities for (2) are obtained by inverting the Miura transformation (see $[12,18]) f=\chi+\varepsilon \chi_{x}-\varepsilon^{2} \chi \mathcal{D} \chi$ from the Gardner's extended $N=1 \operatorname{superKdV}_{\varepsilon}$ equation $[16,17]$

$$
\chi_{t}=\chi_{x x x}+3(\chi \mathcal{D} \chi)_{x}-\frac{1}{2} \varepsilon^{2} \mathcal{D}\left[(\mathcal{D} \chi)^{3}\right]-\frac{3}{2} \varepsilon^{2}\left[\chi(\mathcal{D} \chi)^{2}\right]_{x} .
$$

Recently in [1] it has been observed that the bosonic non-local conserved densities for (1) and (2) are obtained by using an appropriate change of coordinates in the potential superKdV $\mathrm{V}_{\varepsilon}$ equation. Namely, let us introduce the bosonic super-field $\phi(x, t, \theta ; \varepsilon)$ such that $\chi=\mathcal{D} \phi$. Note that the evolution equation for $\phi$ is not in the form of a conserved current,

$$
\phi_{t}=\phi_{x x x}+3 \mathcal{D}\left(\mathcal{D} \phi \cdot \phi_{x}\right)-\frac{1}{2} \varepsilon^{2} \phi_{x}^{3}-\frac{3}{2} \varepsilon^{2} \mathcal{D}\left[\mathcal{D} \phi \cdot \phi_{x}^{2}\right] .
$$

Nevertheless, put $\psi(x, t, \theta ; \varepsilon)=\exp (\varepsilon \phi)$. Then $\psi(x, t, \theta ; \varepsilon)$ satisfies the equation

$$
\psi_{t}=\mathcal{D}\left[\mathcal{D} \psi_{x x}-\frac{3 \psi_{x} \mathcal{D} \psi_{x}}{\psi}+\frac{1}{\varepsilon} \cdot \frac{3 \mathcal{D} \psi \psi_{x}}{\psi}\right]
$$

Potential extended superKdV $\mathrm{V}_{\varepsilon}$ equation $\left(4^{\prime}\right)$ was implicitly described in [1]. We note that equation $\left(4^{\prime}\right)$ is singular in the deformation parameter $\varepsilon$. This is rather unusual with respect to the common practice (see [12] and references therein), although singular transformations did appear [11] in the context of $\varepsilon$-parametric families of integrable equations.

The Taylor coefficients $\psi_{n}$ of the standard decomposition $\psi=\sum_{n=1}^{+\infty} \varepsilon^{n} \cdot \psi_{n}[f]$ are the required [17] nonlocal conserved densities for (2). The transformation $f=f[\psi]$ defines the recurrence relation upon them, and the initial condition is $\psi_{1}=b$. Hence we obtain $\rho_{1}, \rho_{2}$, $\rho_{3}=\frac{1}{6} b^{3}+\mathcal{D}^{-1}(f \mathcal{D} f)$, etc. The new densities $\rho_{n}, n \geq 3$ are still non-local w.r.t. potential superKdV equation (1).

\subsection{Analogues of the $N=1$ super $K d V$ equation}

Now we describe three analogues of superKdV equation (2). These fermionic super-field equations are homogeneous w.r.t. the same weights as (2), and they admit infinitely many higher symmetries proliferated by weakly non-local $[2,22]$ recursion operators. To this end, let the weights $[f]=\frac{3}{2}$ and $[t]=-3$ be fixed. Then we obtain four evolutionary supersymmetric equations (namely, (2) and $(5 \mathrm{a}, \mathrm{b}, \mathrm{c}))$ that admit higher symmetries $f_{s}=\phi$ under the assumption $[s] \geq-5$. The superKdV equation, see (2), is the first in this list. We also get the equation

$$
f_{t}=f_{x x x}+f_{x} \mathcal{D} f
$$

\footnotetext{
${ }^{1}$ Throughout this text, the operator $\mathcal{D}$ acts on the succeeding super-field unless stated otherwise explicitly.
} 
The recursion operator for equation (5a) is constructed in Example 2 on p. 7. Third, we obtain the two-parametric dispersionless analogue of equation (2):

$$
f_{t}=\alpha f \mathcal{D} f_{x}+\beta f_{x} \mathcal{D} f, \quad \alpha, \beta=\text { const } .
$$

A computation by Yu. Naumov (Ivanovo State Power University) with SsTools demonstrates that equation (5b) admits the weakly non-local recursion operator

$$
R=\alpha f \mathcal{D} f \cdot \mathcal{D}+\alpha f f_{x}-\alpha f \mathcal{D} f_{x} \cdot \mathcal{D}^{-1}-\beta f_{x} \mathcal{D} f \cdot \mathcal{D}^{-1}+\beta f_{x} \cdot \mathcal{D}^{-1} \circ(f \mathcal{D}+\mathcal{D} f)
$$

and two infinite sequences of symmetries that start from the translations $f_{x}$ and $f_{t}$.

The fourth equation for the set of weights $[f]=\frac{3}{2},[t]=-3$ is

$$
f_{t}=\mathcal{D}\left(f_{x} f\right) .
$$

It admits the recursion $R=f \mathcal{D}-f_{x} \mathcal{D}^{-1}$, and also it has an infinite sequence of symmetries that starts from the odd weight $[\bar{s}]=-8 \frac{1}{2}$.

Remark 3. The symbols of the evolutionary supersymmetric equations that possess infinitely many symmetries are not necessarily constant. For example, equation (5c) can not be transformed to an equation $g_{t}=g_{x x x}+\cdots$ by a differential substitution $f=f[g]$. The proof is by reductio ad absurdum.

\subsection{Remarks}

In this paper, we investigate the geometric properties of the boson+fermion systems under the additional assumption $[f]=[b]$ (for the primary sets of weights if they are multiply defined). From Axioms 3 and 4 on p. 2 it follows that the triangular systems are regarded as trivial and therefore their properties are not analyzed. We emphasize that, generally, we can not introduce a new anticommuting variable $\Theta$ and then unite the two super-fields $f, b$ to the fermionic superfield $\phi=f+\Theta b$ such that $[\phi]=[f]=[b]-\frac{1}{2}$ or to the bosonic super-field $\beta=b+\Theta f$ such that $[\beta]=[b]=[f]-\frac{1}{2}$ and such that a scalar equation w.r.t. $\phi$ or $\beta$ holds (there is no contradiction with the diagonality assumption because the weights may not be uniquely defined).

We do not expose now the complete list of supersymmetric boson+fermion systems that satisfy the Axioms on p. 2 and such that the weights $[f]=[b]$ coincide. In fact, the symmetries for a major part of these equations are proliferated by the recurrence relations (see p. 8); other equations that admit true recursions seem less physically important than the three variants of the Burgers and the Boussinesq equations we analyze.

Yet it is worthy to note some remarkable features of the five systems such that the weight $[t]=-\frac{1}{2}$ of the time $t$ is half the weight of the spatial variable $x$ (that is, the equations precede the translation invariance). It turned out that these five equations exhibit practically the whole variety of properties that superPDE of mathematical physics possess. Let us briefly summarize these features.

Three of the five evolutionary systems are given through

$$
f_{t}=-\alpha f b, \quad b_{t}=b^{2}+\mathcal{D} f, \quad \alpha=1,2,4 .
$$

The equations differ by the values $\alpha=1,2,4$ of the coefficient and demonstrate different geometrical properties. The geometry of the $\alpha=2$ system is quite extensive: this system admits a continuous sequence of symmetries for all (half-)integer weights $[s] \leq-\frac{1}{2}$, a sequence of symmetries such that the parities of the dependent variables are opposite to the parities of their flows, four local recursions (one is nilpotent), and three local super-recursions. The equation for $\alpha=1$ admits fewer structures, and the case $\alpha=4$ for equation (7) is rather poor. 
Another equation

$$
f_{t}=\mathcal{D} b+f b, \quad b_{t}=\mathcal{D} f
$$

admits local symmetries for all (half-)integer weights $[s] \leq-\frac{1}{2}$. Equation (8) requires introduction of two layers of nonlocalities assigned to (non)local conservation laws. Four nonlocal recursion operators with nonlocal coefficients are then constructed for equation (8). The properties of systems (7), (8) are considered in details in the succeeding paper [7].

The fifth system we mention is a super-field representation of the Burgers equation, see (11); this system is $C$-integrable by using the Cole-Hopf substitution. We investigate its properties in Section 3.

\section{Recursion operators and recurrence relations}

In this section we describe two principally different mechanisms for proliferation of symmetries of a PDE.

\subsection{Differential recursion operators}

We consider the (nonlocal) differential recursion operators first. The standard approach [5, 9] to recursion operators is regarding them as symmetries of the linearized equations. The essence of the method is the following. The 'phantom variables' (the Cartan forms) that satisfy the linearized equation are assigned to all the dependent variables in an equation $\mathcal{E}$; one may think that the internal structure of the symmetries is discarded and the (nonlocal) phantom variables imitate the (resp., nonlocal components of) symmetries for $\mathcal{E}$. In what follows, the capital letters $F, B$, etc. denote the variables associated with the fields $f, b$, respectively. Then any image $\mathcal{R}=R(\varphi)$ of a linear operator $R$ that maps symmetries $\varphi=\left\{f_{s}=F, b_{s}=B\right\}$ of $\mathcal{E}$ to symmetries again is linear w.r.t. the right-hand sides $F, B$. One easily checks that $\mathcal{R}$ is then the r.h.s. of a symmetry flow

$$
\frac{d}{d s_{R}}\left(\begin{array}{l}
F \\
B
\end{array}\right)=\mathcal{R}
$$

for the linearized equation $\operatorname{Lin}(\mathcal{E})$. If the initial equation $\mathcal{E}$ is evolutionary, then the phantom variables satisfy the well-known relations

$$
F_{[t, s]} \doteq 0, \quad B_{[t, s]} \doteq 0
$$

that hold by virtue $(\doteq)$ of the equations $\mathcal{E}$ and $\operatorname{Lin}(\mathcal{E})$. The method is reproduced literally in presence of nonlocalities $w$ whose flows $W$ are described by the corresponding components of nonlocal symmetries $\hat{\varphi}=\left(f_{s}=F, b_{s}=B, w_{s}=W\right)$. The recursion operators are then defined by the triples $\hat{\mathcal{R}}=\left(F_{s_{R}}, B_{s_{R}}, W_{s_{R}}\right)$ and generate sequences of nonlocal symmetries. See [5, 9] for many examples.

We finally recall that not each symmetry $\varphi$ can be extended to a nonlocal flow $\hat{\varphi}$ if the set $\{w\}$ of nonlocalities is already defined and, analogously, not all the pairs $\mathcal{R}=\left(F_{s_{R}}, B_{s_{R}}\right)$ generate a true recursion $\hat{\mathcal{R}}$. The pairs $\mathcal{R}$ are therefore called shadows [9] of nonlocal recursion operators. The shadows are usually sufficient for standard purposes if they describe the operators that map the local components of the flows and whose coefficients are also local. Hence in what follows we always set $W_{s_{R}}=0$ (that is, we do not find the flows $W_{s_{R}}$ that commute with the evolution $W_{t}$ determined by the original system $\mathcal{E}$ and differential substitutions for $w$ ). Also, we describe the Cartan forms $\mathcal{R}$ rather than the differential operators $R$, and we use the term 'recursions' instead of the rigorous 'shadows of the generating Cartan forms for nonlocal recursion operators.' 
Remark 4. The recursion operators considered in this paper are weakly non-local, see [2, 14]. Hence one can readily prove the locality of symmetry sequences generated by these recursions by using a supersymmetric version of the results in [22]. In the sequel, we use another method for the proof of locality. Namely, in Section 4.1.1 we construct a Gardner's integrable deformation [6, $12,18]$ and thus we obtain local Hamiltonian functionals, whence we deduce the locality of the corresponding symmetry flows.

We finally note that a similar method of 'phantom variables' is applied for finding Hamiltonian and symplectic structures for PDE, see [4]. The (nonlocal) Hamiltonian structures for supersymmetrizations (32a), (33) are not extensively studied in this paper. The SsTools package is applicable for this investigation as is, since the theory is now transformed to standard algorithms of symmetry analysis.

Example 2. Consider analogue (5a) of the superKdV equation (2). We introduce the bosonic nonlocality $v$ of weight $[v]=1$ such that $\mathcal{D} v=f$ and the fermionic nonlocality $w$ such that $[w]=\frac{7}{2}$ and $\mathcal{D} w=(\mathcal{D} f)^{2}$. In this setting, we obtain the recursion

$$
\mathcal{R}_{[2]}=\left(\mathcal{D} f \cdot F+3 F_{x x}+f_{x} \cdot V+\frac{1}{2} W\right) .
$$

The above solution generates the sequence $f_{x} \mapsto f_{t} \mapsto \cdots$ of symmetries for equation (5a). The sequence starts with the translation along $x$ and next contains the equation itself.

Next, we consider equation (5b) and introduce two bosonic nonlocal variables $v$ and $w$ such that $\mathcal{D} v=f$ and $\mathcal{D} w=f \mathcal{D} f$. Then we obtain the nonlocal recursion

$$
\mathcal{R}=\alpha \cdot\left(\mathcal{D} f_{x} f V-f \mathcal{D} f \mathcal{D} F+f_{x} f F\right)+\beta \cdot\left(f \mathcal{D} f V-f_{x} W\right) .
$$

The corresponding operator $R$ is present in (6) on p. 5 .

Finally, equation (5c) is obviously a continuity relation. Therefore, we let $v$ be the bosonic variable such that $\mathcal{D} v=f$; hence we obtain the recursion $F_{s_{R}}=f \mathcal{D} F-f_{x} V$.

Remark 5. The systems that admit several scaling symmetries and hence are homogeneous w.r.t. different weights allow to apply the breadth search method for recursions, which is the following. Let a recursion of weight $\left[s_{R}\right]$ w.r.t. a particular set of weights for the super-fields $f, b$ and the time $t$ be known. Now, recalculate its weight $\left[s_{R}^{\prime}\right]$ w.r.t. another set and then find all recursion operators of weight $\left[s_{R}^{\prime}\right]$. The list of solutions will incorporate the known recursion and, possibly, other operators. Generally, their weights will be different from the weight of the original recursion w.r.t. the initial set. Hence we repeat the reasonings for each new operator and thus select the weights $\left[s_{R}\right]$ such that nontrivial recursions exist. This method is a serious instrument for the control of calculations and elimination of errors. We used it while testing the second version of the SsTools package [8].

The second version of SsTools allows to reduce the search for nonlocal recursion operators to solving large overdetermined systems of nonlinear algebraic equations for the undetermined coefficients which are present in the weight-homogeneous ansatz for $F_{s_{R}}, B_{s_{R}}$. The algebraic systems are then solved by using the program CRACK [26]. The nonlocal variables, which are assigned to conservation laws if $N \leq 1$, were also obtained by SsTooLs straightforwardly using the weight homogeneity assumptions.

Remark 6. The weights of the nonlocal variables constructed by using conserved currents for PDE are defined by obvious rules. Clearly, if the weight for a bosonic nonlocality is zero, then further assumptions about the maximal power of this variable in any ansatz should be made. Within this research we observed that the weights of the new super-fields necessary for constructing the recursions are never negative. 


\subsection{Recurrence relations}

Consider an evolution equation $\mathcal{E}=\left\{u_{t}=\phi\right\}$ and let there be a differential function $q[f, b]$ of weight zero w.r.t. an admissible set of weights for $\mathcal{E}$. Suppose further that the flow $u_{s^{n}}=q^{n} \cdot \phi$ is a symmetry of $\mathcal{E}$ for any $n \in \mathbb{N}$; in a typical situation all the flows $\varphi_{n}=u_{s^{n}}$ commute with each other. Then, instead of an infinite sequence $\varphi_{n}$ we have just one right-hand side $u_{s}=Q(q) \cdot \phi$ of fixed differential order and weight $[\phi]$; here $Q$ is an arbitrary analytic function. In this case, we say that the sequence of the Taylor monomials $\varphi_{n}$ is generated by a recurrence relation.

We note that the multiplication by $q$ can be a zero-order recursion operator $R=q$ for the whole symmetry algebra $\operatorname{sym} \mathcal{E}$, otherwise the recurrence relation $\varphi_{n+1}=q \cdot \varphi_{n}$ generates the symmetries of $\mathcal{E}$ for the fixed 'seed' flows $\varphi_{0}$. The systems that admit recurrence relations for their symmetries can possess differential recursion operators as well.

Example 3. Consider the family of supersymmetric systems

$$
f_{t}=b \mathcal{D} b+f \mathcal{D} f, \quad b_{t}=\alpha f \mathcal{D} b,
$$

here $\alpha \in \mathbb{R}$ is arbitrary. We see that equation (9) is homogeneous w.r.t. the weights $[f]=[b]=\frac{1}{2}$, $[t]=-1$. The multiplication of the r.h.s. in (9) by $b$ defines a recurrence relation for infinitely many symmetries. Indeed, the flows

$$
f_{t}=b Q(b) \cdot \mathcal{D} b+f Q(b) \cdot \mathcal{D} f, \quad b_{t}=\alpha f Q(b) \cdot \mathcal{D} b
$$

commute for all $Q$ s and any constant $\alpha$. Nevertheless, the operator $\left(\begin{array}{ll}b & 0 \\ 0 & b\end{array}\right)$ is not a recursion for equation (9) because it does not map an arbitrary symmetry to a symmetry.

Further, let $\alpha=1$. The system

$$
f_{t}=b \mathcal{D} b+f \mathcal{D} f, \quad b_{t}=f \mathcal{D} b
$$

admits the local zero-order recursions

$$
\begin{aligned}
& \mathcal{R}_{\left[1 \frac{1}{2}\right]}^{1}=\left(\begin{array}{c}
\mathcal{D} b b B+\mathcal{D} b f F-\mathcal{D} f f B \\
\mathcal{D} b f B
\end{array}\right), \quad \mathcal{R}_{[2]}^{2}=\left(\begin{array}{c}
b_{x} b F+f_{x} b B \\
b_{x} b B
\end{array}\right), \\
& \mathcal{R}_{[3]}^{3}=\left(\begin{array}{c}
\mathcal{D} b b_{x} b B+\mathcal{D} b b_{x} f F-\mathcal{D} b f_{x} f B-\mathcal{D} f b_{x} f B \\
\mathcal{D} b b_{x} f B
\end{array}\right) .
\end{aligned}
$$

An infinite number of local recursion operators for equation (9a) is obtained by multiplication of $\mathcal{R}^{1}$ by $b^{n}, n \in \mathbb{N}$. The recursions $\mathcal{R}^{1}$ and $\mathcal{R}^{3}$ are nilpotent: $\left(\mathcal{R}_{\left[1 \frac{1}{2}\right]}^{1}\right)^{4}=0=\left(\mathcal{R}_{[3]}^{3}\right)^{4}$.

If $\alpha=-1$, then equation (9) also admits infinitely many symmetries that do not originate from any recurrence relation because their differential orders grow.

The recurrence relation $\varphi_{n+1}\left(\varphi_{n}, q, n\right)$ can depend explicitly on the subscript $n$; then the generators of commuting flows contain the free functional parameters $Q(q), Q^{\prime}(q)$, etc.

Example 4. The flows

$$
\left(\begin{array}{l}
f_{s}(Q) \\
b_{s}(Q)
\end{array}\right)=\left(\begin{array}{c}
\alpha f_{x} Q(b)+\gamma b_{x} f Q^{\prime}(b)+\delta f b^{2} Q^{\prime}(b) \\
\alpha b_{x} Q(b)+\beta f_{x} f Q^{\prime}(b)
\end{array}\right)
$$

commute for arbitrary functions $Q(b)$ and constants $\alpha, \beta, \gamma, \delta \in \mathbb{R}$. Indeed, for any $Q(b)$ and $S(b)$ we have

$$
\left\{\left(\begin{array}{l}
f_{\tau}(Q) \\
b_{\tau}(Q)
\end{array}\right),\left(\begin{array}{l}
f_{\sigma}(S) \\
b_{\sigma}(S)
\end{array}\right)\right\}=0
$$

The flows defined in (10) are also translation and scaling invariant. 
In the sequel, we investigate the systems that are located on the diagonal $[f]=[b]$ and admit infinite sequences of (commuting) symmetries generated by recursion operators; we also analyze generalizations of these equations and properties of the new systems. The supersymmetric equations whose symmetries are proliferated by the recurrence relations are not discussed in this paper.

\section{The Burgers equation}

In this section we investigate three systems related with the Burgers equation. We consider an $N=1$ super-field representation of the Burgers equation and analyze its symmetry properties, we relate a fermionic extension of the Burgers equation with the Burgers equation on associative algebras, and we indicate an $N=2$ scalar super-equation whose $N=1$ diagonal reduction $\left(\theta^{1}=\theta^{2}\right)$ is the bosonic super-field Burgers equation again.

\subsection{Super-field representation for the Burgers equation}

Consider the system

$$
f_{t}=\mathcal{D} b, \quad b_{t}=b^{2}+\mathcal{D} f .
$$

There is a unique set of weights $[f]=[b]=\frac{1}{2},[t]=-\frac{1}{2},[x]=-1$ in this case. Hence we conclude that the above system precedes the invariance w.r.t. the translation along $x$. Equation (11) admits the continuous sequence (14) of higher symmetries $f_{s}=\phi^{f}, b_{s}=\phi^{b}$ at all (half-)integer weights $[s] \leq-\frac{1}{2}$. Also, there is another continuous sequence (15) of symmetries for equation (11) at all (half-)integer weights $[\bar{s}] \leq-\frac{1}{2}$ of the odd 'time' $\bar{s}$.

System (11) is obviously reduced to the bosonic super-field Burgers equation

$$
b_{x}=b_{t t}-2 b b_{t}, \quad b=b(x, t, \theta) .
$$

We emphasize that the role of the independent coordinates $x$ and $t$ is reversed w.r.t. the standard interpretation of $t$ as the time and $x$ as the spatial variable. The Cole-Hopf substitution $b=$ $-u^{-1} u_{t}$ from the heat equation

$$
u_{x}=u_{t t}
$$

provides the solution for the bosonic component of (11).

Further, we introduce the bosonic nonlocality $w(x, t, \theta)$ of weight $[w]=0$ by specifying its derivatives,

$$
\mathcal{D} w=-f, \quad w_{t}=-b ;
$$

the variable $w$ is a potential for both fields $f$ and $b$. The nonlocality satisfies the potential Burgers equation $w_{x}=w_{t t}+w_{t}^{2}$ such that the formula $w=\ln u$ gives the solution; the relation $f=-\mathcal{D} w$ determines the fermionic component in system (11).

Now we extend the set of dependent variables $f, b$, and $w$ by the symmetry generators $F, B$, and $W$ that satisfy the respective linearized relations upon the flows of the initial super-fields. In this setting, we obtain the recursion

$$
\mathcal{R}_{[1]}=\left(\begin{array}{l}
F_{x}-\mathcal{D} f F+f_{x} W \\
B_{x}-\mathcal{D} f B+b_{x} W
\end{array}\right)
$$

of weight $\left[s_{R}\right]=-1$. The operator assigned to $\mathcal{R}$ is

$$
R=\left(\begin{array}{cc}
D_{x}-\mathcal{D} f+f_{x} \mathcal{D}^{-1} & 0 \\
b_{x} \mathcal{D}^{-1} & D_{x}-\mathcal{D} f
\end{array}\right)
$$


The above recursion $R$ is weakly non-local $[2,14]$, that is, each nonlocality $\mathcal{D}^{-1}$ is preceded with a (shadow [9] of a nonlocal) symmetry $\varphi_{\alpha}$ and is followed by the gradient $\psi_{\alpha}$ of a conservation law: $R=$ local part $+\sum_{\alpha} \varphi_{\alpha} \cdot \mathcal{D}^{-1} \circ \psi_{\alpha}$. From [2] it follows that this property is satisfied by all recursion operators which are constructed by using one layer of the nonlocal variables assigned to conservation laws. The weak non-locality of recursion operators is essentially used in the proof of locality of the symmetry hierarchies they generate, see [22] and Remark 4 on p. 7 .

Recursion (13) generates two sequences of higher symmetries for system (11):

$$
\left(\begin{array}{c}
f_{t} \\
b_{t}
\end{array}\right) \mapsto\left(\begin{array}{c}
\mathcal{D} b_{x}-\mathcal{D} f \mathcal{D} b-f_{x} b \\
\mathcal{D} f_{x}-(\mathcal{D} f)^{2}-b^{2} \mathcal{D} f+b b_{x}
\end{array}\right) \mapsto \cdots, \quad\left(\begin{array}{c}
f_{x} \\
b_{x}
\end{array}\right) \mapsto\left(\begin{array}{c}
f_{x x}-2 \mathcal{D} f f_{x} \\
b_{x x}-2 \mathcal{D} f b_{x}
\end{array}\right) \mapsto \cdots
$$

The same recursion (13) produces two infinite sequences of symmetries with the odd parameters $\bar{s}$ for the Burgers equation:

$$
\left(\begin{array}{c}
\mathcal{D} f \\
\mathcal{D} b
\end{array}\right) \mapsto\left(\begin{array}{c}
\mathcal{D} f_{x}-(\mathcal{D} f)^{2}-f_{x} f \\
\mathcal{D} b_{x}-\mathcal{D} f \mathcal{D} b-b_{x} f
\end{array}\right) \mapsto \cdots, \quad\left(\begin{array}{c}
f \mathcal{D} b-b \mathcal{D} f+b_{x} \\
b \mathcal{D} b-f \mathcal{D} f+f_{x}-f b^{2}
\end{array}\right) \mapsto \cdots
$$

Remark 7. System (11) is not a supersymmetric extension of equation (12); it is a representation of the bosonic super-field Burgers equation. The flows in (14) become purely bosonic in the coordinates $c=\mathcal{D} f, b$. The standard recursion for the Burgers equation, see (20) on p. 12, acts 'across' the two sequences in (14) and maps $\left(f_{t}, b_{t}\right) \mapsto\left(f_{x}, b_{x}\right)$; again, we note that the independent coordinates in (12) are reversed w.r.t. (19). Surprisingly, the flow that succeeds the translation along $x$ in (14) reappears in (17).

However, from the above reasonings we profit two sequences of symmetries (15), which are not reduced to the bosonic $(x, t)$-independent symmetries $[10, \S 8.2]$ of the Burgers equation. We finally recall that the Burgers equation (12) has infinitely many higher symmetries that depend explicitly on the base coordinates $x, t$ but exceed the set of Axioms on p. 2.

\subsection{Supersymmetric extension of the Burgers equation}

The fermionic extension of the Burgers equation,

$$
f_{t}=f_{x x}+(b f)_{x}, \quad b_{t}=b_{x x}+b b_{x}+\alpha f_{x} f, \quad \alpha \in \mathbb{R}
$$

is a unique extension of the Burgers equation that admits higher symmetries and which was found by using SsTools. It must be noted that equation (16) contains the unknowns $f(x, t), b(x, t)$, and it seems to have nothing to do with a supersymmetry. The one-parametric family (16) admits the symmetries $\left(\begin{array}{l}f_{s} \\ b_{s}\end{array}\right)$ at all negative integer weights $[s] \leq-1$. We also note that system (16) is the Burgers equation itself if the fermionic field $f(x, t)$ is set to zero.

In what follows, we distinguish the two cases: $\alpha=0$ and $\alpha \in \mathbb{R} \backslash\{0\}$ in equation (16). We claim that the algebraic properties of corresponding extensions (16) for the Burgers equation are then different. Indeed, system (16) is triangular if the coupling constant $\alpha$ equals zero, see Remark 8 below.

Obviously, the weights $[b]=1$ are $[t]=-2$ are fixed in system (16). If $\alpha=0$, then the weight $[f]$ of the fermionic field is arbitrary since the first equation in (16) is linear w.r.t. the derivatives of $f$. If $\alpha \neq 0$, then the weights of the field $f(x, t)$ and of the dimensional real constant $\alpha$ are related by the formula $[f]=1-\frac{1}{2}[\alpha]$, see Remark 2 on p. 3 for discussion. In particular, we have $[f]=1$ if $\alpha$ is a true constant w.r.t. the scalings and hence its weight equals

zero. We conclude that the fields $b$ and $f$ can not be coupled to the super-field $u=b+\vartheta f$ using a super-variable $\vartheta$ unless $[f]=\frac{3}{2}$ and an additional relation unites the weights of $\vartheta$ and $\alpha$. In the sequel, we consider both cases: $[f]=\frac{3}{2}$ and $[f] \neq \frac{3}{2}$. 
The fermionic component in (16) is linear w.r.t. the field $f(x, t)$, and hence the superposition principle is valid for it. The quantity

$$
\int_{-\infty}^{+\infty} f(x, t) \mathrm{d} x=\text { const }
$$

is an integral of motion for (16). The fermionic variable $w(x, t)$ is assigned to the conserved current $D_{t}(f)=D_{x}\left(f_{x}+b f\right)$ : we set $w_{x}=f$ such that $w \cdot w=0$ and $[w]=0$.

Remark 8. System (16) models an unusual physical phenomenon. Assume that at any point $x \in \mathbb{R}$ there are two types of a physical value, the bosonic field with density $b(x, t)$ and the "invisible" fermionic field with density $w(x, t)$, and let the dynamics of these two fields be described by system (16) (or equation (17) below). Suppose that the initial numeric values $w(x, 0)$ and $b(x, 0)$ of the fermionic and bosonic densities, respectively, coincide at $t=0$. If $\alpha=0$, then densities will coincide for all $t>0$ and the corresponding integral quantities $\int_{-\infty}^{+\infty} w(x, t) \mathrm{d} x$ and $\int_{-\infty}^{+\infty} b(x, t) \mathrm{d} x$ will be conserved in time.

If $\alpha \neq 0$, then the feed-back is switched on in (16). A ripple in the fermionic space is the cause for the bosonic integral $\int_{-\infty}^{+\infty} b(x, t) \mathrm{d} x$ to change. Indeed, this quantity is no longer conserved unless $w(x, t)=$ const or, generally, unless the condition $\int_{-\infty}^{+\infty} w_{x x}(x, t) w_{x}(x, t) \mathrm{d} x=$ const holds for all $t>0$. We see that the reaction of the fermionic component on the bosonic field depends on the incline $w_{x}=f$ and curvature $w_{x x}=f_{x}$ but not on the density $w(x, t)$.

\subsubsection{Trivial coupling in (16): $\alpha=0$}

Let us suppose that $\alpha=0$. Then from (16) we obtain the system

$$
w_{t}=w_{x x}+b w_{x}, \quad b_{t}=b_{x x}+b b_{x} .
$$

System (17) appeared in [23] and [25] in a different context: both fields $w(x, t)$ and $b(x, t)$ were regarded as bosonic, and then Bäcklund autotransformations [25] and the linearizing substitutions [23] were constructed. In Remark 7 we noted that the two-component bosonic system (17) originates from a flow in (14). The field $w(x, t)$ was recognized as fermionic in [3], where the Painlevé properties of (17) and related systems were investigated. In what follows, the field $w(x, t)$ is a fermionic dependent variable.

Remark 9. The bosonic component $b(x, t)$ in $(17)$ is linearized by the Cole-Hopf substitution

$$
b=2 q^{-1} q_{x}
$$

where the function $q(x, t)$ is a solution of the heat equation $q_{t}=q_{x x}$. From (18) it follows that the Burgers equation is the factor of the heat equation w.r.t. its scaling symmetry. This scheme is of general nature and can be used for constructing new equations from homogeneous systems. In this paper we do not investigate the relation between the scaling invariance of superPDE and their factorizations w.r.t. the scaling symmetries, and we do not study the physical significance of these projectivizations and of the new equations.

Now we reduce (16) for $\alpha=0$ to one scalar super-equation w.r.t. a new field which is constructed as follows. Let $\vartheta$ be the new independent super-variable such that

- its square vanishes, $\vartheta \cdot \vartheta=0$;

- the variable $\vartheta$ anticommutes with the fermionic field $f$ and its derivatives with respect to $x$;

- the weight of $\vartheta$ is $[\vartheta]=-\frac{1}{2}$.

Recall that the weight of the field $f$ is not fixed if $\alpha=0$. Hence we postulate $[f]=\frac{3}{2}$. 
Further, we define the bosonic field $u=b+\vartheta f$ of weight $1=[b]=[\vartheta]+[f]$. Then we obtain the Burgers super-field equation again! Indeed, we have

$$
u_{t}=u_{x x}+u u_{x} .
$$

However, we note that the super-derivative $D_{\vartheta}+\vartheta D_{x}$ whose square is $D_{x}$ does not appear in the reasonings. Therefore, the weights are motivated by the background concept of supersymmetry, but they are not uniquely defined. One could easily fix $[f]:=1$ and $\vartheta:=0$ with the same equation (19) in the end.

Equation (19) has the well-known recursion operator

$$
R=D_{x}+\frac{1}{2} u+\frac{1}{2} u_{x} D_{x}^{-1} \Longleftrightarrow \mathcal{R}=U_{x}+\frac{1}{2} u U+\frac{1}{2} u_{x} D_{x}^{-1}(U) .
$$

The recursion operators for the left- and right-noncommutative $\star$-Burgers equations have been obtained by M. Gürses and A. Karasu (private communication).

Let us construct an analogue of recursion (20) for system (16) if the coupling is $\alpha=0$. First we introduce the bosonic nonlocality $v(x, t)$ of weight zero by setting $v_{x}=b$. The potentials $v$ and $w$ satisfy the system

$$
w_{t}=w_{x x}+v_{x} w_{x}, \quad v_{t}=v_{x x}+\frac{1}{2} v_{x}^{2} .
$$

Next, we make a technical assumption that the bosonic variables $v$ and $V$ of zero weight appear in the recursion for (16) at most linearly. Then we find out that in this setting there are two recursion operators of weight 1 . The first operator,

$$
\mathcal{R}_{[1]}^{1}=\left(\begin{array}{c}
-\frac{1}{2} w B_{x}+\frac{1}{2} b_{x} W-\frac{1}{4} b_{x} w V+F_{x}+\frac{1}{2} f_{x} V+\frac{1}{2} b F-\frac{1}{4} w b B-\frac{1}{4} f b V \\
2 B_{x}+b B+b_{x} V
\end{array}\right),
$$

is the direct extension of twice the recursion (20) for equation (19). Simultaneously, we obtain the shadow recursion with nonlocal coefficients,

$$
\mathcal{R}_{[1]}^{2}=\left(\begin{array}{c}
w B_{x}+b_{x} W+\frac{1}{2} b_{x} w V+2 F_{x}+f_{x} V+b F+\frac{1}{2} w b B+\frac{1}{2} f b V+2 f B \\
0
\end{array}\right) .
$$

Its differential order is 1 ; this recursion is not nilpotent.

\subsubsection{Arbitrary coupling in (16): $\alpha \in \mathbb{R}$}

In this subsection we consider the case of an arbitrary non-zero real constant $\alpha$ in system (16).

In view of the preceding subsection, we introduce the independent variable $\vartheta$ that anticommutes with the fermionic field $f$ and its derivatives w.r.t. $x$ and such that

- $\vartheta \cdot \vartheta=\alpha$, that is, the square of $\vartheta$ is now non-zero;

- the weights of the new variable $\vartheta$, the dimensional constant $\alpha$ (see Remark 2), and the fermionic field $f$ are related by the formulas $2[\vartheta]=[\alpha],[f]=1-[\vartheta]$.

This time we set $[\vartheta]=[\alpha]:=0,[f]:=1$, and we do not consider the square root of $D_{x}$ based on the variable $\vartheta$. We emphasize that $\vartheta$ is not an ordinary complex number whose square could easily be zero, negative, or positive (and $\vartheta$ would therefore be zero, imaginary, or negative, respectively).

Again, we set $u=b+\vartheta f$; the field $u(x, t ; \vartheta)$ is homogeneous of weight 1 . Moreover, it satisfies the Burgers equation

$$
u_{t}=u_{x x}+u u_{x},
$$


but now equation $\left(19^{\prime}\right)$ is an equation on the associative algebra generated by $u$ and its derivatives w.r.t. $x$. This is because for all $i \neq j$ we have ${ }^{2}\left[u_{i}, u_{j}\right] \sim \alpha \neq 0$; for example,

$$
u u_{x}=b b_{x}+\vartheta^{2} f_{x} f+\vartheta \cdot\left(b_{x} f+b f_{x}\right) \neq b b_{x}-\vartheta^{2} f_{x} f+\vartheta \cdot\left(b_{x} f+b f_{x}\right)=u_{x} u .
$$

The geometry of equations on associative algebras has been studied recently, see [20], by using standard notions and computational algorithms.

Obviously, the fermionic nonlocality $w$ such that $w_{x}=f$ is indifferent w.r.t. the value of the coupling constant $\alpha$. We find out that system (16) always admits the conservation law that potentiates the bosonic variable $b$. We thus set

$$
\tilde{v}_{x}=b+\frac{1}{2} f w \alpha, \quad \tilde{v}_{t}=b_{x}+\frac{1}{2} b^{2}+\frac{1}{2} f_{x} w \alpha+\frac{1}{2} f b w \alpha .
$$

The weight of the nonlocality $\tilde{v}$ is zero. Surprisingly, the variable $\tilde{v}$ satisfies the same potential Burgers equation as the nonlocality $v$, see equation (21),

$$
w_{t}=w_{x x}+\tilde{v}_{x} w_{x}, \quad \tilde{v}_{t}=\tilde{v}_{x x}+\frac{1}{2} \tilde{v}_{x}^{2} .
$$

We conclude that equation $\left(21^{\prime}\right)$ potentiates system (16) for all $\alpha=\vartheta \cdot \vartheta$, although the algebraic nature of the Burgers equation (19) w.r.t. the field $u=b+\vartheta f$ is radically different from equation $\left(19^{\prime}\right)$ that describes the Burgers flow on the associative algebra.

In the nonlocal setting $\{f, w\}+\{b, \tilde{v}\}$ there are two generalizations of recursion (20). The first recursion of weight 1 for equation (11) is

$$
\mathcal{R}_{[1]}^{1}=\left(\begin{array}{c}
b_{x} W+2 F_{x}+f_{x} \tilde{V}+\frac{1}{2} f_{x} w W \alpha+b F+f B \\
2 B_{x}+b B+b_{x} \tilde{V}+\frac{1}{2} b_{x} w W \alpha+f_{x} W \alpha-f F \alpha
\end{array}\right),
$$

here we underline the component that corresponds to (20). The second extension of weight 1 is

$$
\begin{aligned}
\mathcal{R}_{[1]}^{2, f}= & -w B_{x}-\frac{1}{2} b_{x} w \tilde{V}+\frac{1}{2} f_{x} w W \alpha-\frac{1}{4} w f b W \alpha-\frac{1}{2} w f F \alpha-\frac{1}{2} w b B-\frac{1}{2} f b \tilde{V}-f B, \\
\mathcal{R}_{[1]}^{2, b}= & \frac{2 B_{x}+b B+b_{x} \tilde{V}}{}-w F_{x} \alpha+f_{x} W \alpha+\frac{1}{2} f_{x} w \tilde{V}+\frac{1}{2} f b W \alpha-\frac{1}{2} w b F \alpha \\
& -\frac{1}{2} w f b \tilde{V} \alpha-\frac{3}{2} w f B \alpha .
\end{aligned}
$$

Remark 10. In the previous reasonings we treated system (16) as an $N=0$ equation that involves the fermionic field but does not contain the super-derivative $\mathcal{D}$. Now we enlarge the $(x, t, f, b)$ jet space with the anticommuting independent variable $\theta$ and the derivatives of $f$ and $b$ w.r.t. $\theta$. We have $\mathcal{D}^{2}=D_{x}$, and the unknown functions become the super-fields $f(x, t, \theta)$ and $b(x, t, \theta)$. Physically speaking, we permit the consideration of conservation laws at halfinteger weights for (16) and $\left(21^{\prime}\right)$. We discover that there are many nonlocal conservation laws for equation (16); for example, we obtain the 'square roots' of the variables $\tilde{v}$ and $w$. We conjecture that there are infinitely many $N=1$ conservation laws for equation (11). Also, there are many recursions that involve the nonlocalities assigned to the new conservation laws and which are nilpotent if $\alpha=0$. This situation is analogous to the scheme that generates non-local Hamiltonians for the $N=1$ superKdV equation (2), see p. 4 and $[1,17]$.

\section{3 $N=2$ supersymmetric Burgers equation}

Let us recall that $D_{\theta^{i}}$ and $D_{x}$ denote the derivatives w.r.t. the independent coordinates $\theta^{i}$ and $x$, respectively, while $\mathcal{D}_{i}$ are the super-derivations such that $\mathcal{D}_{i}^{2}=D_{x}$ for any $i$.

We now admit that Axiom 8 was used when constructing the evolutionary super-systems:

\footnotetext{
${ }^{2}$ One could use the notation $\hbar$ instead of $\alpha$.
} 
8. Each of the super-derivatives $\mathcal{D}_{i}=D_{\theta^{i}}+\theta^{i} D_{x}, i=1, \ldots, N$, occurs at least once in the r.h.s. of the evolutionary system if $N \geq 2$.

In the database [27] there is a scalar, third order $N=2$ supersymmetrization of the Burgers equation; it is

$$
b_{t}=\mathcal{D}_{1} \mathcal{D}_{2} b_{x}+b b_{x}, \quad b=b\left(x, t, \theta^{1}, \theta^{2}\right), \quad \mathcal{D}_{i}=D_{\theta^{i}}+\theta^{i} D_{x}, \quad i=1,2 .
$$

Equation (22) is reduced to the second order Burgers super-field equation $b_{t}=b_{x x}+b b_{x}$ on the super-diagonal $\theta^{1}=\theta^{2}$, here $b=b\left(t, x, \theta^{1}, \theta^{1}\right)$.

Let us expand the super-field $b\left(x, t ; \theta^{1}, \theta^{2}\right)$ in $\theta^{1}$ and $\theta^{2}$ :

$$
b=\beta(x, t)+\theta^{1} \xi(x, t)+\theta^{2} \eta(x, t)+\theta^{1} \theta^{2} \gamma(x, t) .
$$

Hence from equation (22) we obtain the system for the components of $b$ :

$$
\begin{aligned}
& \beta_{t}=-\gamma_{x}+\beta \beta_{x}, \quad \xi_{t}=\eta_{x x}+(\beta \xi)_{x}, \quad \eta_{t}=-\xi_{x x}+(\beta \eta)_{x}, \\
& \gamma_{t}=\beta_{x x x}+(\beta \gamma)_{x}-(\xi \eta)_{x} .
\end{aligned}
$$

We see that the second and third equations in system $\left(22^{\prime}\right)$ are Burgers-type, that is, they contain the dissipative terms and the remaining parts are total derivatives. The fourth equation in $\left(22^{\prime}\right)$ describing the evolution of $\gamma$ is of KdV-type: the dispersion and two divergent terms are present in it.

The KdV nature of the $N=2$ supersymmetric Burgers equation (22) is not occasional. Indeed, equation (22) is a symmetry of the $N=2$ supersymmetric $\mathrm{SKdV}_{4}$ equation [13]

$$
b_{s}=-b_{x x x}+\frac{1}{2}\left(b \mathcal{D}_{1} \mathcal{D}_{2} b\right)_{x}+\frac{3}{4}\left(\mathcal{D}_{1} b \mathcal{D}_{2} b\right)_{x}+\frac{3}{4} b^{2} b_{x} .
$$

Reciprocally, the $\mathrm{SKdV}_{4}$ equation (23) is a higher symmetry of the Burgers equation (22), and their bi-Hamiltonian hierarchy is of the form

$$
b_{x} \mapsto \text { equation }(22) \mapsto \text { equation }(23) \mapsto \cdots \text {. }
$$

The two equations share the recursion operator $[13,17]$. It must be noted that the relation between the Laberge-Mathieu's $N=2 \mathrm{SKdV}_{4}$ equation and the $N=2$ Burgers system was not indicated in loc. cit.

\section{The Boussinesq equation}

In this section we describe a super-field representation of the dispersionless Boussinesq equation; the system at hand admits two infinite sequences of commuting symmetries of constant differential order 2 which are generated by a weakly non-local recursion operator of differential order 1. Also, we extend the Boussinesq system with dispersion and dissipation to its threeparametric analogue that does not retract to it for any values of the parameters.

\subsection{The dispersionless Boussinesq equation}

We consider the two-component system

$$
f_{t}=b \mathcal{D} b, \quad b_{t}=\mathcal{D} f_{x} .
$$

System (24) is a super-representation of the dispersionless Boussinesq equation

$$
b_{t t}=\frac{1}{2}\left(b^{2}\right)_{x x},
$$

here $b(x, t, \theta)$ is the bosonic super-field. In what follows, we construct a Gardner's deformation for the hydrodynamic representation of the dispersionless Boussinesq equation (25). Next, we transmit the properties of Hamiltonian symmetries for equation (25) onto its supersymmetric representation (24). 


\subsubsection{The Gardner's deformation of the dispersionless Boussinesq equation}

The bosonic two-component form of $(25)$ is

$$
b_{t}=c_{x}, \quad c_{t}=b b_{x} .
$$

Here we obviously have $c(x, t ; \theta)=\mathcal{D} f(x, t ; \theta)$. System $(26)$ is a super-field equation of hydrodynamic type.

Remark 11. Consider the dispersionless Boussinesq equation (26) with $b(x, t)$ and $c(x, t)$. The number of independent variables in it coincides with the number of unknown functions and equals two. Therefore, the system at hand is linearized [21] by using the hodograph transformation $b(x, t), c(x, t) \mapsto x(b, c), t(b, c)$. Indeed, we obtain the linear autonomous system

$$
x_{c}=t_{b}, \quad t_{c}=\frac{x_{b}}{b},
$$

here $c$ is the new time and $b$ is the new spatial variable. The solution of the hydrodynamic type system $\left(26^{\prime}\right)$ is expressed with the Airy function.

Now we construct a Gardner's deformation [6, 12, 18] for system (26). We have

$$
\left(\begin{array}{l}
b \\
c
\end{array}\right)_{t}=\left(\begin{array}{cc}
0 & D_{x} \\
D_{x} & 0
\end{array}\right)\left[\begin{array}{l}
\delta / \delta b \\
\delta / \delta c
\end{array}\right]_{(x, t)} \mathcal{H}(b, c)
$$

where the density of the Hamiltonian $\mathcal{H}$ is $H=\frac{1}{6} b^{3}+\frac{1}{2} c^{2}$. We proceed with a deformation

$$
\left(\begin{array}{l}
w^{1} \\
w^{2}
\end{array}\right)_{t}=\left(\begin{array}{cc}
0 & D_{x} \\
D_{x} & 0
\end{array}\right)\left[\begin{array}{l}
\delta / \delta w^{1} \\
\delta / \delta w^{2}
\end{array}\right]_{(x, t)} \overline{\mathcal{H}}\left(w^{1}, w^{2} ; \varepsilon\right)
$$

of equation $\left(26^{\prime \prime}\right)$. We assume that the deformations of the dependent variables are

$$
b=w^{1}+\varepsilon W_{1}(\boldsymbol{w})+\varepsilon^{2} W_{2}(\boldsymbol{w})+\cdots, \quad c=w^{2}+\varepsilon \Omega_{1}(\boldsymbol{w})+\varepsilon^{2} \Omega_{2}(\boldsymbol{w})+\cdots,
$$

and we extend the Hamiltonian functional such that its density is

$$
\bar{H}=H(\boldsymbol{w})+\varepsilon H_{1}(\boldsymbol{w})+\varepsilon^{2} H_{2}(\boldsymbol{w})+\cdots .
$$

We also expand the fields $w^{1}(x, t ; \varepsilon)$ and $w^{2}(x, t ; \varepsilon)$ in $\varepsilon$ :

$$
w^{i}(x, t ; \varepsilon)=\sum_{k=0}^{+\infty} w_{k}^{i} \varepsilon^{k}, \quad i=1,2 .
$$

Recall that equation (26) is in the divergent form. Therefore, the Taylor coefficients $w_{k}^{i}$ are termwise conserved, and from (28a) we get the initial conditions $w_{0}^{1}=b, w_{0}^{2}=c$ and the recurrence relations for the conserved densities.

Now we truncate expansions (28a), (28b) to polynomials of sufficiently large degrees. By using the homogeneity reasonings, in view of $[\varepsilon]=-3$, we then reduce the deformation problem to an algebraic system for the undetermined coefficients in these expansions. We solve the algebraic system by using the program CRACK [26] and finally obtain the deformation

$$
\begin{aligned}
& b=w^{1}+\varepsilon w^{1} w^{2}, \\
& c=w^{2}+\frac{1}{3} \varepsilon\left(w^{1}\right)^{3}+\varepsilon\left(w^{2}\right)^{2}+\frac{1}{3} \varepsilon^{2}\left(w^{2}\right)^{3}, \\
& \bar{H}=\frac{1}{6}\left(w^{1}\right)^{3}+\frac{1}{2}\left(w^{2}\right)^{2}+\frac{1}{6} \varepsilon\left(w^{2}\right)^{3} .
\end{aligned}
$$


The initial terms in the two sequences of the conserved densities are

$$
\begin{array}{lll}
w_{0}^{1}=b, & w_{1}^{1}=-b c, & w_{2}^{1}=2 b c^{2}+\frac{1}{3} b^{4}, \quad \ldots, \\
w_{0}^{2}=c, & w_{1}^{2}=-c^{2}-\frac{1}{3} b^{3}, & w_{2}^{2}=\frac{5}{3} c^{3}+\frac{5}{3} b^{3} c, \quad \ldots
\end{array}
$$

Taking into account that the Hamiltonian operator $\left(\begin{array}{cc}0 & D_{x} \\ D_{x} & 0\end{array}\right)$ maps gradients of conservation laws for equation (26) to its symmetries, see [9], we thus conclude that (29) describes two infinite sequences of Hamiltonians for (26). They determine infinitely many contact symmetry flows, which are local w.r.t. $b$ and $c$. Moreover, each flow lies in the image of $D_{x}$ by construction, and hence they induce local symmetry transformations of the super-variable $f(x, t ; \theta)$ such that $c=\mathcal{D} f$. We emphasize that we did not even need a recursion operator for (26) to obtain the contact symmetry flows and prove their locality.

Remark 12. The problem of constructing integrable deformations for homogeneous (super-) PDE is another practical application of the CRACK solver [26] for large overdetermined systems of nonlinear algebraic equation. This application has not been previously considered within the framework of $[7,26,27]$.

\subsubsection{Supersymmetric representation of equation (25)}

Now we discuss the Hamiltonian properties of system (24). It is homogeneous w.r.t. multiply defined weights; we let the primary set be $[f]=[b]=1,[t]=-1 \frac{1}{2},[x]=-1$. With respect to this set, system (24) admits two infinite sequences of Hamiltonian symmetry flows of unbounded weights $-1,-1 \frac{1}{2},-2 \frac{1}{2},-3, \ldots$, and constant differential order 2 . These two sequences start with the flows

$$
\left(\begin{array}{l}
f_{x} \\
b_{x}
\end{array}\right) \mapsto\left(\begin{array}{l}
\mathcal{D} b b^{2}+\mathcal{D} f f_{x} \\
\mathcal{D} f_{x} b+\mathcal{D} f b_{x}
\end{array}\right) \mapsto \cdots, \quad\left(\begin{array}{l}
f_{t} \\
b_{t}
\end{array}\right) \mapsto\left(\begin{array}{l}
\mathcal{D} f \mathcal{D} b b+\frac{1}{2} f_{x} b^{2} \\
\mathcal{D} f_{x} \mathcal{D} f+\frac{1}{2} b_{x} b^{2}
\end{array}\right) \mapsto \cdots
$$

The skew-adjoint operator $A=\left(\begin{array}{cc}0 & \mathcal{D} \\ -\mathcal{D} & 0\end{array}\right)$ is a Hamiltonian structure for the symmetries in $(30)$, and, by Section 4.1.1, all the flows possess the Hamiltonian functionals. There are two Casimirs $H_{0}^{(1)}=b, H_{0}^{(2)}=\mathcal{D} f$ for equation (24). From (29) we obtain the Hamiltonians with densities

$$
\begin{aligned}
& H_{1}^{(1)}=b \mathcal{D} f, \quad H_{2}^{(1)}=\frac{1}{12} b^{4}+\frac{1}{2} b(\mathcal{D} f)^{2}, \quad \ldots, \\
& H_{1}^{(2)}=\frac{1}{2}(\mathcal{D} f)^{2}+\frac{1}{6} b^{3}, \quad H_{2}^{(2)}=\frac{1}{6}(\mathcal{D} f)^{3}+\frac{1}{6} b^{3} \mathcal{D} f, \quad \ldots
\end{aligned}
$$

Let us construct the nonlocal recursion that maps the symmetries in (30). To this end, we introduce the nonlocality $w$ such that $w_{t}=b^{2} / 2$ and $\mathcal{D} w=f$. We further let the variable $v$ be such that $v_{t}=\mathcal{D} f, v_{x}=b$. Then we obtain the nonlocal recursion

$$
\mathcal{R}_{\left[1 \frac{1}{2}\right]}=\left(\begin{array}{l}
\mathcal{D} b b V+\frac{1}{2} b^{2} \mathcal{D} V+\frac{3}{4} \mathcal{D} f F+\frac{3}{4} f_{x} W \\
\mathcal{D} f_{x} V+\frac{1}{2} b \underline{\mathcal{D} F}+\frac{3}{4} \mathcal{D} f B+\frac{3}{4} b_{x} W
\end{array}\right)
$$

of differential order 1 . Indeed, the order of the flow $b_{s_{i+1}}$ equals the order of $f_{s_{i}}$ plus 1 owing to the presence of the underlined differential operator $\mathcal{D}=D_{\theta}+\theta D_{x}$ in $\mathcal{R}^{b}$.

Proposition. Recursion (31) proliferates the symmetries of constant differential order 2 for the dispersionless Boussinesq super-field equation (24). The initial terms of the symmetry sequences are given in (30), and their Hamiltonians are $\left(29^{\prime}\right)$.

The assertion follows from the results of Section 4.1.1.

We note that Hamiltonian symmetries (30) are an example of infinitely many flows that are not obtained by a recurrence multiplication scheme, see p. 8 for definition, although their differential order is constant. Indeed, they are contact in the coordinates $b, c$ and are of second order w.r.t. $f$ and $b$. 


\subsection{The Boussinesq equation with dispersion and dissipation}

Representation (24) of the dispersionless Boussinesq super-field equation is embedded in the one-parametric family of supersymmetric systems

$$
f_{t}=b \mathcal{D} b+\mathcal{D} b_{x x}-\alpha f_{x x}, \quad b_{t}=\mathcal{D} f_{x}+\alpha b_{x x}, \quad \alpha \in \mathbb{R} .
$$

By definition, put $c=\mathcal{D} f$. Then from (32a) we get the bosonic Boussinesq system (see, e.g., $[6,12,19])$

$$
c_{t}=b b_{x}+b_{x x x}-\alpha c_{x x}, \quad b_{t}=c_{x}+\alpha b_{x x}, \quad \alpha \in \mathbb{R} .
$$

The two systems (32) are homogeneous w.r.t. the weights $[b]=2,[f]=2 \frac{1}{2},[c]=3$, and $[t]=-2$. We see that equation (32a) has no nontrivial bosonic limit. Indeed, one can not set $f \equiv 0$ such that the fermionic equation remains consistent unless $b=$ const.

If $\alpha=0$, then (32) is the Boussinesq equation with dispersion. The terms involving $\alpha$ describe the dissipation. There is a well-known recursion operator of weight $\left[s_{R}\right]=-3$ for the Boussinesq equation without dissipation [19]. The recursion for the fermionic component in (32a) is then, roughly speaking, the component corresponding to $c$ in the recursion for (32b) conjugated by $\mathcal{D}$.

If $\alpha$ is non-zero, then system (32b) is not reduced to a scalar fourth order super-field equation. In this case, the system is translation invariant and for all $\alpha \in \mathbb{R}$ it admits symmetries of weights $[s]=-\left(3 k+\frac{3}{2} \pm \frac{1}{2}\right), k \in \mathbb{N}, k \geq 0$.

\subsection{The multi-parametric Boussinesq-type equation}

Finally, we construct the Boussinesq-type system using representation (32a) for the Boussinesq equation. From the database [27] we obtain the system

$$
\begin{aligned}
& f_{t}=\alpha \beta f b-\alpha \gamma b \mathcal{D} b-\gamma^{2} \mathcal{D} b_{x x}-\beta \gamma f_{x x}, \\
& b_{t}=\alpha \beta b^{2}+\beta^{2} \mathcal{D} f_{x}+\beta \gamma b_{x x}
\end{aligned}
$$

here $\alpha, \beta, \gamma \in \mathbb{R}$. This system is an analogue of Boussinesq equation (32) but does not retract to it for any value of the parameters. Similarly to equation (32a), system (33) does not have a nontrivial bosonic limit at $f \equiv 0$.

The translation invariance of equation (33) is obvious; we recall that $[x]=-1$ and $[t]=-2$. Also, equation (33) admits the symmetry

$$
\begin{aligned}
f_{s}= & -\mathcal{D} b b_{x x} \gamma^{3}+\mathcal{D} b_{x} b_{x} \gamma^{3}+\mathcal{D} b_{x} \mathcal{D} f \beta \gamma^{2}-\mathcal{D} f_{x} \mathcal{D} b \beta \gamma^{2}-\mathcal{D} f_{x} f \beta^{2} \gamma+\mathcal{D} f f_{x} \beta^{2} \gamma \\
& -b_{x x} f \beta \gamma^{2}+b_{x} f_{x} \beta \gamma^{2}, \\
b_{s}= & -\mathcal{D} b f_{x} \beta^{2} \gamma+\mathcal{D} b_{x} f \beta^{2} \gamma+\mathcal{D} b_{x} \mathcal{D} b \beta \gamma^{2}+f_{x} f \beta^{3}
\end{aligned}
$$

such that $[s]=-4$, and there is the symmetry

$$
\begin{aligned}
& f_{\bar{s}}=\mathcal{D} b f_{x} \beta \gamma^{2}-\mathcal{D} b_{x} f \beta \gamma^{2}-\mathcal{D} b_{x} \mathcal{D} b \gamma^{3}-b_{x}^{2} \gamma^{3}-(\mathcal{D} f)^{2} \beta^{2} \gamma-f_{x} f \beta^{2} \gamma-2 \mathcal{D} f b_{x} \beta \gamma^{2}, \\
& b_{\bar{s}}=\mathcal{D} b b_{x} \beta \gamma^{2}+\mathcal{D} f \mathcal{D} b \beta^{2} \gamma+\mathcal{D} f f \beta^{2} \gamma+b_{x} f \beta^{2} \gamma,
\end{aligned}
$$

here the weight of the odd parameter $\bar{s}$ is $[\bar{s}]=-3 \frac{1}{2}$. No more symmetries exist for equation (33) if $[s] \geq-7$ and if the parity of $s$ is arbitrary. Quite strangely, there are no conservation laws for equation (33) within the weights not greater than 11; hence no nonlocalities were constructed and no recursion is currently known for system (33). 


\section{Conclusion}

In this paper, we investigated the integrability of fermionic extensions and supersymmetric generalizations for the KdV, Burgers, and Boussinesq equations. Recursion operators for their symmetry algebras were obtained. Also, we analyzed the properties of Gardner's deformations for the dispersionless Boussinesq equation (26) and $N=1$ supersymmetric KdV equation (2).

The boson+fermion super-field representations (11), (24), and (32) for the Burgers and Boussinesq equations are remarkable by themselves. Indeed, the roles of the independent variables $x$ and $t$ are swapped in system (11): $x$ is the time and $t$ is the spatial coordinate in equation (12). The dispersionless Boussinesq system (24) admits two recursive sequences (30) of Hamiltonian symmetries whose differential orders is constant, while the differential order of nonlocal recursion (31) is strictly positive. The Boussinesq-type system (33) is multi-parametric and contains the Boussinesq equation with dispersion as a component but can not be reduced to it at any values of the parameters. We finally note that all the Boussinesq-type systems (24), (32a), (33), as well as representation (11) for the Burgers equation, do not have bosonic limits at $f \equiv 0$.

Using fermionic extension (16) of the Burgers equation, we conclude that the 'direct $N \geq 1$ supersymmetrization' [16] based on replacing the derivatives with super-derivatives in a PDE is not a unique way to obtain its generalizations. Indeed, the new systems can contain the fermionic fields but no super-derivatives. Hence we indicate three possible types of these generalizations.

1. The new super-fields combine the old fields with the new added components and satisfy manifestly $N \geq 1$ supersymmetric equations, see the superKdV equation (2), for example.

2. The evolution of new fermionic fields $f(x, t)$ is coupled with the original equation, see (16). Then, new independent (Grassmann, or super-)variables $\vartheta$ are introduced such that the extended system is reduced to a smaller equation. The geometric structures of the extension are then inherited from the final system by routine expansions in the new variables $\vartheta$, see (19).

3. Similarly to the previous case, the new field is constructed by using a new independent variable that anticommutes with the fermionic function but not with itself. Then the resulting system is an evolution equation on an associative algebra [20], see equation $\left(19^{\prime}\right)$ on p. 12.

We conclude that the equations on associative algebras present a nontrivial way to generalize coupled fermion+boson systems.

\section{Acknowledgements}

The authors thank V.V. Sokolov for formulation of the classification problem and also thank A.V. Mikhailov, A. Sergyeyev, and A.S. Sorin for helpful discussions. The authors are greatly indebted to the referees for their remarks and suggestions. T.W. wishes to thank W. Neun for discussions and the SHARCNET consortia for computer access. The research of A.K. was partially supported by the Scientific and Technological Research Council of Turkey (TUBITAK).

A part of this research was carried out while A.K. was visiting at Brock University and at Middle East Technical University (Ankara).

[1] Andrea S., Restuccia A., Sotomayor A., The Gardner category and nonlocal conservation laws for $N=1$ Super KdV, J. Math. Phys., 2005, V.46, 103513, 11 pages; hep-th/0504149.

[2] Bilge A.H., On the equivalence of linearization and formal symmetries as integrability tests for evolution equations, J. Phys. A: Math. Gen., 1993, V.26, 7511-7519.

[3] Hlavatý L., The Painlevé analysis of fermionic extensions of KdV and Burgers equations, Phys. Lett. A, 1989, V.137, N 4-5, 173-178. 
[4] Kersten P., Krasil'shchik I., Verbovetsky A., Hamiltonian operators and $\ell^{*}$-coverings, J. Geom. Phys., 2004, V.50, N 1-4, 273-302; math.DG/0304245.

[5] Kersten P., Krasil'shchik I., Verbovetsky A., (Non)local Hamiltonian and symplectic structures, recursions and hierarchies: a new approach and applications to the $N=1$ supersymmetric KdV equation, J. Phys. A: Math. Gen., 2004, V.37, 5003-5019; nlin.SI/0305026.

[6] Kiselev A.V., Karasu A., Hamiltonian deformations of the Boussinesq equations, Proc. Workshop 'Quantization, Dualities, and Integrable Systems' (January 23-27, 2006, Denizli, Turkey), Preprint ISPUmath-1/2006, 12 pages.

[7] Kiselev A.V., Wolf T., On weakly non-local, nilpotent, and super-recursion operators for $N=1$ homogeneous super-equations, Proc. Int. Workshop 'Supersymmetries and Quantum Symmetries - 2005' (July 26-31, 2005, Dubna, Russia), to appear; nlin.SI/0511056.

[8] Kiselev A.V., Wolf T., The SsTools environment for classification of integrable super-equations, Comp. Phys. Commun., 2006, to appear.

[9] Krasil'shchik I.S., Kersten P.H.M., Symmetries and recursion operators for classical and supersymmetric differential equations, Dordrecht, Kluwer Acad. Publ., 2000.

[10] Krasil'shchik I.S., Lychagin V.V., Vinogradov A.M., Geometry of jet spaces and nonlinear partial differential equations, New York, Gordon \& Breach Sci. Publ., 1986.

[11] Kupershmidt B.A., Singular symmetries of integrable curves and surfaces, J. Math. Phys., 1982, V.23, 364-366.

[12] Kupershmidt B.A., Deformations of integrable systems, Proc. Roy. Irish Acad. A, 1983, V.83, 45-74.

[13] Laberge C.A., Mathieu P., $N=2$ superconformal algebra and integrable $O(2)$ fermionic extensions of the Korteweg-de Vries equation, Phys. Lett. B, 1988, V.215, 718-722.

[14] Maltsev A.Ya., Novikov S.P., On the local systems Hamiltonian in the weakly non-local Poisson brackets, Phys. D, 2001, V.156, 53-80; nlin.SI/0006030.

[15] Manin Yu.I., Radul A.O., A supersymmetric extension of the Kadomtsev-Petviashvili hierarchy, Comm. Math. Phys., 1985, V.98, 65-77.

[16] Mathieu P., Supersymmetric extension of the Korteweg-de Vries equation, J. Math. Phys., 1988, V.29, 2499-2506.

[17] Mathieu P., Open problems for the super KdV equations. Bäcklund and Darboux transformations. The geometry of solitons, CRM Proc. Lecture Notes, 2001, V.29, 325-334; math-ph/0005007.

[18] Miura R.M., Gardner C.S., Kruskal M.D., Korteweg-de Vries equation and generalizations. II. Existence of conservation laws and constants of motion, J. Math. Phys., 1968, V.9, 1204-1209.

[19] Olver P.J. Applications of Lie groups to differential equations, 2nd ed., New York, Springer-Verlag, 1993.

[20] Olver P.J., Sokolov V.V., Integrable evolution equations on associative algebras, Comm. Math. Phys., 1998, V.193, 245-268.

Olver P.J., Sokolov V.V., Non-abelian integrable systems of the derivative nonlinear Schrödinger type, Inverse Problems, 1998, V.14, L5-L8.

[21] Roždestvenskiŭ B.L., Janenko N.N., Systems of quasilinear equations and their applicatons to gas dynamics, Translations of Mathematical Monographs, Vol. 55, Providence, RI, AMS, 1983.

[22] Sergyeyev A., Locality of symmetries generated by nonhereditary, inhomogeneous, and time-dependent recursion operators: a new application for formal symmetries, Acta Appl. Math., 2004, V.83, 95-109; nlin.SI/0303033.

Sergyeyev A., Why nonlocal recursion operators produce local symmetries: new results and applications, J. Phys. A: Math. Gen., 2005, V.38, 3397-3407; nlin.SI/0410049.

[23] Svinolupov S.I., On the analogues of the Burgers equation, Phys. Lett. A, 1989, V.135, 32-36.

[24] Tsuchida T., Wolf T., Classification of polynomial integrable systems of mixed scalar and vector evolution equations. I, J. Phys. A: Math. Gen., 2005, V.38, 7691-7733; nlin.SI/0412003.

[25] Weiss J., Tabor M., Carnevale G., The Painlevé property for partial differential equations, J. Math. Phys., 1983, V.24, 522-526.

[26] Wolf T., Applications of CRACK in the classification of integrable systems, CRM Proc. Lecture Notes, 2004, V.37, 283-300; nlin.SI/0301032.

[27] Wolf T., Supersymmetric evolutionary equations with higher order symmetries, 2003, http://beowulf.ac.brocku.ca/ twolf/htdocs/susy/all.html (please contact T. W. for access). 